\title{
Alcohol's effects during uncertain and uncontrollable stressors in the laboratory.
}

Daniel E. Bradford ${ }^{1,2}$, Jack M. Shireman ${ }^{3}$, Sarah J. Sant'Ana ${ }^{4}$, Gaylen E. Fronk ${ }^{4}$, Susan E.

$$
\text { Schneck }^{4} \text {, John J. Curtin }{ }^{4}
$$

1. Department of Psychology, University of Miami, Coral Gables, Florida, USA

2. School of Psychological Sciences, Oregon State University, Corvallis, Oregon, USA

3. Neuroscience Training Program, University of Wisconsin, Madison, Wisconsin, USA 4. Department of Psychology, University of Wisconsin, Madison, Wisconsin, USA

\section{Corresponding Author:}

Daniel E. Bradford, Department of Psychology, University of Miami

5665 Ponce De Leon Blvd. Coral Gables, Florida 33146-2510

E-mail: dbradford@miami.edu

Phone: (305) 284-8057

Preprint: This article may not exactly replicate the final version published in the journal. It is not the copy of record.

This article was accepted to the journal Clinical Psychological Science on September 14, 2021. 


\begin{abstract}
Alcohol's effects on reactivity to stressors depends on the nature of the stressor and the reactivity being assessed. Research identifying characteristics of stressors that modulate reactivity and clarifies the neurobehavioral, cognitive, and affective components of this reactivity may help prevent, reduce or treat the negative impacts of acute and chronic alcohol use with implications for other psychopathology involving maladaptive reactivity to stressors. We used a novel, multi-measure, cued electric shock stressor paradigm in a greater university community sample of adult recreational drinkers to test how alcohol $(\mathrm{N}=64)$, compared to No-alcohol ( $N=64)$, effects reactivity to stressors that vary in both their perceived certainty and controllability. Preregistered analyses suggested alcohol significantly dampened subjective anxiety (self-report) and defensive reactivity (startle potentiation) more during uncertain than during certain stressors regardless of controllability, suggesting that stressor uncertainty — but not uncontrollability — may be sufficient to enhance alcohol's stress reactivity dampening and thus negative reinforcement potential.
\end{abstract}


For millennia, humans have used drugs such as alcohol to avoid or alleviate the detrimental effects of psychological stressors (Hanson, 2013; Sher, 1987). Frequent, excessive use of alcohol when "drinking to cope" with some stressors may eventually lead to exaggerated stressor reactivity, which may cause, maintain, or exacerbate alcohol use disorder and other psychopathology (Kaye et al., 2017; Koob \& Volkow, 2010). Different types of stressors modulate reactivity in different ways and alcohol may not diminish reactivity to all stressors equally (Sayette, 2017). Research that both identifies key characteristics of stressors that modulate reactivity and clarifies the neurobehavioral, cognitive, and affective components of this reactivity may help prevent, reduce or treat the negative impacts of acute and chronic alcohol use behavior. This research may also guide similar efforts with other psychopathology involving maladaptive reactivity to stressors (e.g., pathological anxiety and depression; Grillon, 2008; Havranek et al., 2016; Insel et al., 2010; Maier, 2015).

Researchers have identified several distinct characteristics of stressors that modulate reactivity (Davis et al., 2010; Kaye et al., 2017; Maier, 2015; Zvolensky et al., 2000). In particular, parallel but separate lines of evidence indicate that stressors that are either uncertain or uncontrollable elicit greater reactivity and have more detrimental effects on physical health and cognitive performance than their certain and controllable counterparts (Henderson et al., 2012; Koolhaas et al., 2011; Mineka \& Kihlstrom, 1978; Robinson et al., 2013). Characteristics of certainty and controllability have also emerged as important in the etiology and/or manifestations of some mood and anxiety disorders. Specifically, patients with either Post Traumatic Stress Disorder or Panic Disorder display exaggerated reactivity selectively to uncertain (relative to certain) stressors (Grillon et al., 2008; Grillon, Pine, et al., 2009). In parallel, exposure to uncontrollable (but not controllable) stressors has figured prominently in 
etiologic models of depression such as "Learned Helplessness" in animals and "Learned Hopelessness" in humans (Abramson et al., 1978; Liu et al., 2015).

Recent research has also focused specifically on reactivity to uncertain stressors to clarify the negative reinforcing effects of anxiolytic medications and popular recreational yet addictive drugs such as alcohol (Davis et al., 2010; Kaye et al., 2017; Maier, 2015). Commonly prescribed anxiolytics (e.g., benzodiazepines, SSRIs) appear to reduce reflexive defensive reactivity (i.e., startle potentiation) to a greater degree when experimentally manipulated stressors (e.g., cued threat of electric shock) are presented with uncertainty (e.g., unpredictable timing) rather than with certainty (e.g., predictable timing; Grillon, Chavis, et al., 2009; Grillon et al., 2006). Similarly, a programmatic series of experiments in our laboratory has demonstrated that single administrations of moderate doses of alcohol (e.g., dose yielding blood alcohol concentrations; BACs; between $0.08-0.15 \%$ ) temporarily reduce startle potentiation during uncertain stressors significantly more than certain stressors (Bradford et al., 2013, 2017; Hefner et al., 2013; Hefner \& Curtin, 2012; Moberg \& Curtin, 2009). Furthermore, Kaye et al. (2017) have proposed that chronic use of alcohol causes etiologically relevant stress neuroadaptations (see Koob \& Volkow, 2010) that manifest as persistent exaggerated reactivity to uncertain stressors in patients with alcohol use disorder. Moberg et al. (2017) provided preliminary support for this thesis (see also Hefner et al., 2018; Hogle et al., 2010 for comparable exaggerated uncertain stressor reactivity in chronic marijuana and tobacco users, respectively).

It appears that stressors play an important role in the reinforcing effects of alcohol and other drugs and the etiology or manifestations of psychopathology involving addiction, anxiety and depression. Furthermore, characteristics of these stressors that alter their certainty and controllability are crucial to consider as we clarify their impact. Critically, at least four important 
gaps remain in our understanding of these phenomena. First, research on stressor certainty and controllability typically focuses on only one of these characteristics, ignoring or even confounding the other (e.g., uncertain stressors are also uncontrollable in most experiments). This has prevented unambiguous identification of either the unique or interactive effects of each characteristic. Second, modern methods have largely focused on a single measure of a specific response domain (e.g., defensive reactivity). However, measures of defensive reactivity such as startle potentiation are affected by both affect and attention (Blumenthal, 2015). To assess clearly and comprehensively the multiple mechanisms affected by different stressor characteristics, a single study must use multiple measures ${ }^{1}$. Third, although Maier and others (e.g., Maier, 2015; Maier \& Watkins, 2005) have conducted an elegant series of studies explicitly examining the effects of uncontrollable and controllable stressor exposure with animals, stressor controllability research using animal-to-human translational methods has only begun to be used in humans (Havranek et al., 2016; Löw et al., 2015; Wendt et al., 2017). Finally, although the effects of alcohol and other drugs on responses to uncertain stressors has

\footnotetext{
1 "In this study, we use startle potentiation and probe P3 suppression to index defensive reactivity and emotionally motivated attention, respectively. Eye-blink startle is a cross-species brainstem-controlled response elicited by, for example, sudden and loud auditory "startle probes". Believed to have evolved as part of an organism's bodily defense against threats, this response is made larger in magnitude (i.e., "startle potentiation") when sub-nuclei in the extended amygdala are activated in the presence of a threatening stimulus such as visual cues that signal threat of electric shock (Grillon, 2008). Furthermore, evidence suggests that startle potentiation during uncertain vs certain threats is governed by overlapping but dissociable brain structures (Davis et al., 2010). This positions startle potentiation during uncertain and certain threats as an important translational measure that allows for inference about neural mechanisms involved with response to various stressor types and the effects of drugs thereon (Kaye et al., 2017). Sudden auditory stimuli such as the startle probe used to elicit the startle response also evoke a frequently studied brain wave ERP response known as the P3 (see Linden, 2005 for review). The magnitude of the P3 appears to reflect attentional processing with a larger P3 to a stimulus suggesting more attentional resources devoted to processing it. As stressors and other emotionally evocative stimuli demand an organism's limited attentional resources (Blanchard et al., 2011; Robinson et al., 2015), the P3 to the benign auditory startle probe is subsequently attenuated (i.e., probe $\mathrm{P} 3$ suppression) in the presence of more threatening stimuli such as visual cues signaling threat of shock. We (Bradford, et al., 2017) and others (Nelson et al., 2015) have used probe P3 suppression as "relatively pure index" (Bradley et al., 2006) of emotionally motivated attention in experimental stressor paradigms that also include measurement of startle potentiation."
} 
received recent attention, no study to date has tested the effects of a pharmacological manipulation (e.g., alcohol) on responses to uncontrollable and controllable stressors.

These gaps leave three possibilities, each with unique implications about understanding of the effects of drugs on reactivity to stressors and that reactivity itself: 1) Alcohol's effects on stressor reactivity are limited to stressors that are both uncertain and uncontrollable, suggesting a synergistic process across two stressor dimensions with implications for both the effects of alcohol and how stressor characteristics interact to affect reactivity; 2) Alcohol reduces stressor reactivity more during uncertain (vs. certain) stressors and also more during uncontrollable (vs. controllable) stressors, providing preliminary evidence for either distinct or shared drug-sensitive mechanisms for reactivity to both stressor types; 3) Alcohol selectively affects uncertain stressor reactivity without varying by stressor controllability, providing an important boundary condition on the circumstances in which alcohol dampens stressor reactivity as well as additional evidence that human reactions to stressor certainty and controllability rely on distinct psychological and/or neurological mechanisms (Davis et al., 2010; Maier \& Watkins, 2005; Wood et al., 2015). We present here the first attempt to discern between these possibilities by using a novel experimental stressor paradigm to test the effects of a moderate dose of alcohol (target BAC $=.08 \%$ ) on selfreported anxiety (subjective anxiety potentiation), defensive reactivity (startle potentiation), and emotionally motivated attention (suppression of the P3 Event Related Potential response to the startle probe, i.e., probe P3 ERP suppression; see Bradford et al., 2017 for a review of these measures) in a factorial 2 (uncertain, certain) X 2 (uncontrollable, controllable) fully crossed design.

\section{Methods}

\section{Open Science and Reproducibility}


We preregistered our sample size determination, data processing parameters. and analysis plan at https://osf.io/q8d96/register/565fb3678c5e4a66b5582f67\#q4. Following recommendations about research transparency (Simmons et al., 2012), we have reported how we determined our sample size, all data exclusions, all manipulations, and all measures in the study. Following emerging open science guidelines (Schönbrodt et al., 2015), we will make curated data and analysis scripts publicly available via OSF at https://osf.io/2zmxe/ upon publication.

\section{Participants}

The study was approved by an Institutional Review Board's Social and Behavioral Sciences human subjects committee, all procedures were carried out in accordance with the provisions of the Declaration of Helsinki, and informed written consent was obtained from all study participants. We recruited a final sample of 128 participants (64 female; see Table 1 for demographics) from the university community. Power analyses using the pwr (Champely, 2020) package in $\mathrm{R}$ ( $\mathrm{R}$ Development Core Team, 2015) indicated that this sample size would provide at least $80 \%$ power to detect the median effect size (partial eta-squared $=.07$ ) observed across previous studies examining alcohol's effects on startle potentiation during uncertain versus certain stressors (e.g., Bradford et al., 2013).

Participants reported that they could read and write in English, were between 21 and 35 years old, had experience within the last year with drinking the quantities of alcohol needed to obtain the study dose of alcohol, reported no history of alcohol-related problems, no current psychiatric medication use, no uncorrected hearing or vision problems, no alcohol contraindicated medical condition, were not pregnant (verified by urine sample), and were sober on arrival (verified via breathalyzer (Alcosensor IV; Intoximeters Inc., St. Louis, MO). We paid participants $\$ 10 / \mathrm{h}$ for their participation. 
Total N

Female (\%)

Non-White (\%)

Hispanic (\%)

\section{Alcohol}

Highest level of education (\%)

High school or equivalent degree Some college or 2-year degree College or Advanced Degree

Annual Income (\%)

less than 20K/year 20-50k/year 50-100k+/year Did not respond

Age
$24.0(3.2)$

Range $=21-33$
64

50

22

5

\section{No-Alcohol}

64

50

16

8

5

$30 \quad 37$

$65-58$

$59-63$

$29 \quad 25$

$11 \quad 11$

$1 \quad 1$

Current alcohol use

$\begin{array}{rll}\text { Frequency (occasions/week) } & 2.3(1.7) & 2.0(1.4) \\ \text { Quantity (per occasion) } & 3.9(2.2) & 4.2(2.4) \\ \text { Past year alcohol problems } & 3.8(2.1) & 3.7(2.1)\end{array}$

Table 1. Demographics (mean and standard deviation unless otherwise noted) for full sample $(N=128)$. Self-reported alcohol problems were measured with the Young Adult Alcohol Problems Scale (Hurlbut \& Sher, 1992). 


\section{Beverage manipulation}

Beverage manipulation procedures were consistent with our laboratory’s standards (e.g., Bradford et al., 2015). After a baseline assessment of general startle reactivity (see Supplemental Materials), we randomly assigned participants to one of three beverage conditions: alcohol $(\mathrm{N}=$ 64), no-alcohol/told alcohol (i.e., placebo; $\mathrm{N}=32$ ) and no-alcohol/told no-alcohol (i.e., true noalcohol; $\mathrm{N}=32$; see Figure 1 for study flow diagram). Expectancies and other cognitions about alcohol and drinking may affect attentional measures (Testa et al., 2006) so we included separate placebo and true no-alcohol conditions to allow exploratory analysis of placebo effects. Past research suggests, however, that most of alcohol's effects on affective responding to noxious stimuli, when present, appear to have a pharmacological basis (see Greeley \& Oei, 1999 for review). Furthermore, we have not found placebo effects in our past research on alcohol and threat uncertainty (Hefner et al., 2013), so our preregistered analyses focused on the contrast between the alcohol group and the combination of the two no-alcohol conditions (i.e., equal $\mathrm{N}$ alcohol and no-alcohol groups). We provide more detail about the alcohol manipulation and results comparing placebo and true no-alcohol conditions for each dependent variable in the Supplemental Materials.

We divided beverages in all three conditions into two drinks, each consumed over 15 min, for a 30 min total drinking period. We began the experimental session approximately 15 min after the end of the drinking period. We measured BAC via breathalyzer (Alcosensor IV; Intoximeters Inc., St. Louis, MO) immediately before and after completion of the main shockthreat task. 


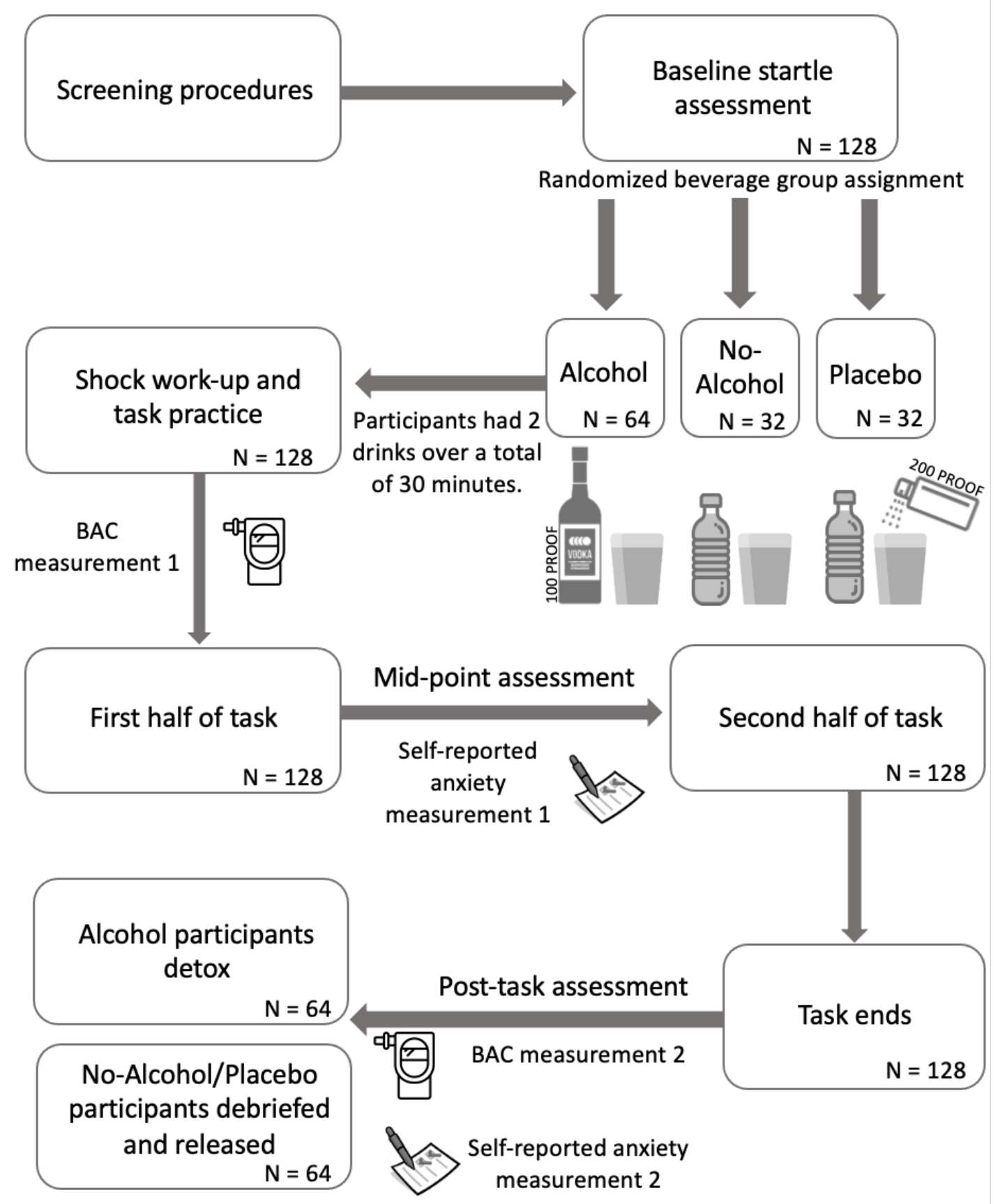

Figure 1. Study flow diagram.

Note: Self-report individual measures were completed after post-task assessment for all participants.

Figure @ 2021 Daniel E. Bradford, Jack M. Shireman, Sarah J. Sant’Ana, Gaylen E. Fronk, Susan E.

Schneck, John J. Curtin under Creative Commons Attribution 4.0 International Public License CC-By. 


\section{Shock work-up procedures}

After the beverage manipulation, we measured participants' subjective tolerance to a series of increasing intensity $200 \mathrm{~ms}$ electric shocks (7 mA maximum) delivered with a custom shock stimulator across the distal phalanges of the index and ring fingers of their non-dominant hand, following standard procedures from our laboratory (Bradford et al., 2014). We set maximum shock intensity during the main task to each participant's subjective maximum tolerance threshold to minimize individual differences in shock tolerance.

\section{Task design}

A PC-based Matlab script written with Psychophysics Toolbox (Brainard, 1997; Pelli, 1997) presented visual and auditory stimuli. Participants viewed serially presented grayscale square visual cues on a CRT monitor for $6 \mathrm{~s}$ each, separated by a variable ITI (Inter-Trial Interval; range 9-15 s, M=12 s). A black text in the center of each visual cue indicated impending shock intensity. Visual cues were presented in within-subject blocks with the same visual cue repeated three times within each block. There were four shock block types: certain and uncontrollable, uncertain and uncontrollable, certain but controllable, and uncertain but controllable as well as one no-shock block type (see Figure 2). Each shock block type was presented twice ( 6 cue presentations) and the no-shock block was presented three times (9 cue presentations) for a grand total of 11 blocks (33 cue presentations) presented in one of 4 counterbalanced orders.

For controllable blocks, we gave participants perceived control of a range of shock intensities opposed to, for example, control of shock occurrence or duration, so uncertain shock trials could remain relatively uncertain even after being lowered (i.e. controlled). This design 
also allowed matching of number and average intensity of shocks received across block types to control for possible interactions of alcohol with intensity of the stress response (e.g., Moberg et al., 2011). Across all certain block cues, participants received shocks equal to $60 \%$ of their maximum tolerated shock intensity (12 shocks total). Across all uncertain block cues, participants received a range of shocks: two shocks each at $20 \%, 40 \%, 80 \%$, and $100 \%$ of their maximum tolerated shock intensity and four shocks at $60 \%$ intensity (mean intensity $=60 \% ; 12$ shocks total). This range was chosen, via pilot testing, to provide easily discernable intensities while ensuring the lowest intensity was still aversive. Shock levels were pseudorandomized within each uncertain block with each beginning with a mean intensity (60\%) shock to avoid interference with the control illusion that could have been caused by beginning with a higher intensity shock on controllable blocks.

We told participants they could receive 10 different shock intensities across blocks. In each of the two certain block types, the impending shock intensity was indicated by a specific number (e.g., 6) displayed in black in the center of each cue. In each of the two uncertain block types, participants were told a range of possible shock values prior to block start (e.g., 4-8) but the exact impending shock intensity for each cue was unknown, and this was indicated by a black question mark in the center of the cue. We told participants they could lower the intensity of the shocks in certain blocks that were also deemed controllable (i.e., certain but controllable) by "two full levels" (e.g., 8 could be lowered to 6). Similarly, participants were told they could lower the range of possible shock intensities in uncertain blocks that were also deemed controllable (i.e., uncertain but controllable) by two full levels (e.g., 6-10 could be lowered to 48). We told participants they could not affect the shock intensity in either of the uncontrollable blocks (i.e., uncertain and uncontrollable; certain and uncontrollable). In all shock blocks, 
participants were aware that the 6 s shock cues would co-terminate with $200 \mathrm{~ms}$ shocks at the indicated intensity (i.e., shocks administered 5.8 seconds post-cue onset).

\section{Perceived control manipulation}

We told participants they could control the shocks during controllable blocks using a custom made, fixed-position, joystick with an on/off rocker switch and spring-loaded trigger. We instructed participants to use the rocker switch to turn on or off their ability to control the shocks prior to the start of controllable and uncontrollable shock blocks, respectively. Participants were then verbally asked: "Are you controlling the shocks now?" to which participants were directed to respond simply "yes" or "no" after they flipped the switch on or off. The switch controlled a custom light box illuminating the words "Shock Control ON" or "Shock Control OFF" positioned in front of the participant directly below the computer monitor. The rocker switch was also wired to an input channel on the data collection computer in the experimenter control room to allow researchers to verify that the participant had properly used the switch. Regardless of the rocker switch position, the shock controller had no real effect on actual shock intensity, as shock intensities were pre-determined as described above.

To control for possible effects of motor movement or attentional effects on the startle or probe $\mathrm{P} 3$ responses, we instructed participants to quickly pull the trigger on the joystick after each cue was presented across all blocks regardless of shock controllability. We told participants to do this to show that they were paying attention during the task. Participants had the entire 6 seconds of the cue presentation to pull the trigger. The stimulus presentation computer recorded trigger pulls. On screen instructions reminded participants to pull the trigger between every block. We discarded and replaced the data from 3 participants who failed to pull the trigger in time on a certain but controllable trial because this undermined the illusion of control. Data were 
not discarded if participants failed to pull the trigger on an uncertain but controllable trial (1 participant) because the shock level remained uncertain on these trials. When participants pulled the trigger, the black text turned grey (on all trials) and shrunk in size (on controllable trials) to indicate that their trigger pull was recorded, and that their control was effective. See Supplemental Materials for a narrative summary of the presentation of each block type separately. 


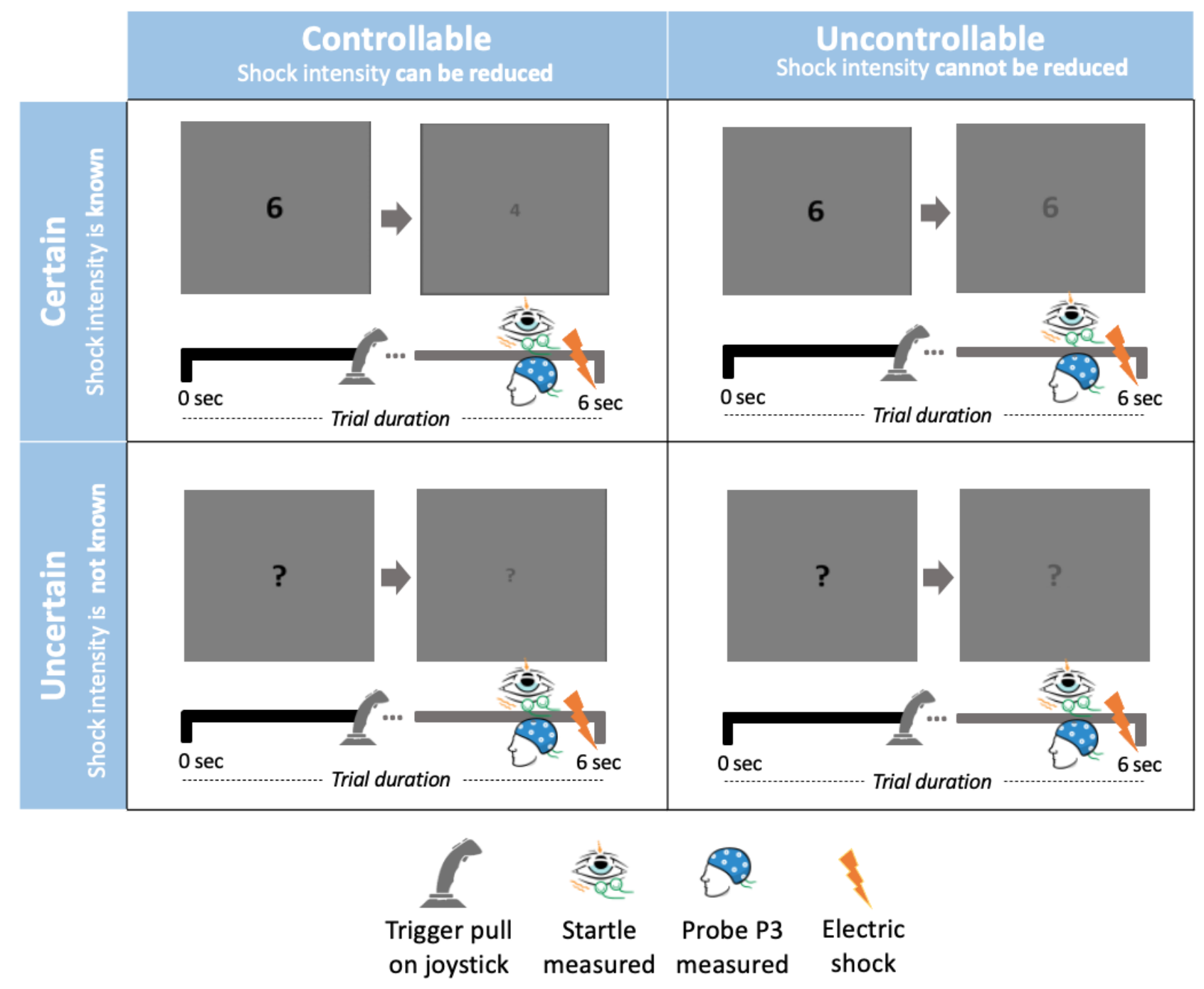

Figure 2. Typical trial timeline

Note: Each shock block type was presented twice ( 3 cues in each block). Three no-shock blocks ( 3 cues in each block) with a black crosshair that turned gray upon trigger pull were also presented (not shown). Inter-trial intervals consisted of a black cross hair and no square (not shown). EMG startle and Probe P3 ERPs were measured at 5.5 seconds into the cue presentation. Shocks occurred at 5.8 seconds and coterminated with cue offset at 6 seconds. Self-reported anxiety to each cue type was measured at a break in the middle and at the end of the task (not shown). Figure (C) 2021 Daniel E. Bradford, Jack M. Shireman, Sarah J. Sant'Ana, Gaylen E. Fronk, Susan E. Schneck, John J. Curtin under Creative Commons Attribution 4.0 International Public License CC-By. 


\section{Mid- and post-task measures}

During a short break at the midpoint and after the main task, participants rated how anxious/fearful they were on a 5-point rating scale ( $1=$ 'not at all anxious/fearful'; $5=$ 'extremely anxious/fearful') when they saw each cue type. After the post task anxiety ratings, participants completed a placebo manipulation check and a battery of self-report individual difference questionnaires (see Supplementary Materials), were debriefed, compensated, and dismissed once reaching a BAC below $0.03 \%$.

\section{Startle response measurement and processing}

We measured the eye-blink startle response to acoustic startle probes $(50 \mathrm{~ms}, 102 \mathrm{~dB}$ white noise with near instantaneous rise time). We presented 6 noise probes during a subset of the visual cues at $5.3 \mathrm{~s}$ post cue onset timing during the baseline procedure and 22 noise probes during a subset of the cues in the threat of shock (16 probes; 4 for each cue type) and no-shock (6 probes) blocks at $5.3 \mathrm{~s}$, post cue onset during the main task. We conducted offline data processing for all physiological signals using the PhysBox plugin (Curtin, 2011) within the EEGLab toolbox (Delorme \& Makeig, 2004) in MATLAB (MATLAB and Statistics Toolbox, 2013). We followed our preregistration and published guidelines (Blumenthal et al., 2005; Bradford et al., 2014) for startle response reduction and processing (See Supplemental Materials for more detail).

\section{Probe P3 measurement and processing}

We sampled the EEG signal at $2500 \mathrm{~Hz}$ from 3 midline scalp sites (Fz, Cz, Pz,). We measured the P3 ERP to the acoustic startle probes at electrode site Pz, consistent with our preregistration and recent published reports (Alius et al., 2015; Bradford et al., 2017; Nelson \& 
Hajcak, 2017). Data from the two other electrode sites were collected for goals not relevant to the current study. EEG response reduction and processing followed published guidelines (Picton et al., 2000) and our preregistration (see Supplemental Materials for more detail).

\section{Analysis Plan}

Our analysis followed our preregistration plan and previous research (e.g., Bradford et al., 2013, 2017). We calculated general startle reactivity as mean startle response to startle probes during cues in the baseline procedure, startle potentiation as the mean increase in startle response to probes during cues in the shock blocks relative to cues in the no-shock blocks, subjective anxiety potentiation as the mean increase in anxiety to the shock cues relative to the no-shock cues, and probe $\mathrm{P} 3$ suppression as the mean decrease in the P3 ERP to startle probes during cues in the shock blocks relative to cues in the no-shock blocks.

We analyzed each dependent variable in separate, General Linear Models (GLMs) using RStudio (RStudio Team, 2016) for R with the ImSupport (Curtin, 2015) package. All GLMs included between-subjects regressors for beverage group (alcohol vs placebo vs true no-alcohol) and within-subjects factors for shock certainty (uncertain minus certain) and shock

controllability (uncontrollable minus controllable). Beverage group was coded with two planned orthogonal contrasts to examine effects of alcohol (alcohol group vs the no-alcohol participants (i.e., combination of the placebo and true no-alcohol groups)) and the placebo manipulation (placebo group vs true no-alcohol group). All a priori hypotheses concerning alcohol were interpreted using the alcohol contrast (no-alcohol participants as reference), as preregistered. We tested the effects of controllability in the no-alcohol participants as a manipulation check. Though not preregistered, we added additional tests of the effects of certainty (in the no-alcohol participants) and the main effects of alcohol as additional manipulation checks for completeness 
and clarity. In the Supplemental Materials, we report placebo effects (true no-alcohol as reference) and the preregistered description of covariate selection methods.

To address our substantive research questions, we tested for a three-way, alcohol X certainty X controllability interaction for each dependent variable. Following our preregistration, in the presence of a significant three-way interaction, we tested 3 planned contrasts with comparing the certain and controllable reference condition with: 1 . Startle potentiation in uncontrollable and uncertain, 2. Startle potentiation in uncontrollable and certain, 3. Startle potentiation in controllable and uncertain. In absence of a significant three-way interaction, we tested the two, two-way alcohol X certainty and alcohol X controllability interactions as preregistered. For transparency, we report all predicted means and standard errors from GLMs for each individual threat-type variant by beverage group in the Supplemental Materials.

We used our field's standard $p<.05$ criteria for determining that results from tests were significantly different from expected if data were consistent with the null hypothesis. We report both partial eta-squared $\left(\eta_{p}{ }^{2}\right)$ and GLM coefficients $(b)$ to describe effect sizes. We conducted outlier analysis (studentized residual with Bonferroni corrected $p<.05$ ) in preliminary GLMs for each dependent variable. This resulted in the removal, without replacement, of 2, 8, and 1 outliers for GLMs for subjective anxiety potentiation, startle potentiation, and probe P3 suppression, respectively.

\section{Results}

The mean BAC for participants administered alcohol was $0.07 \%(S D=0.01 \%)$ immediately before the main task, and $0.07 \%(S D=0.01 \%)$ immediately after the task. Consistent with our previous work, the placebo manipulation was successful in establishing 
expectations of alcohol consumption and intoxication (see Supplemental Materials for full analysis of the placebo manipulation and reliability of all measures).

In the no-alcohol participants, subjective anxiety potentiation was significantly greater during uncertain than certain threats, $\eta_{p}^{2}=.42, b=.5, t(115)=9.10, p<.001$ (Figure 3A). Startle potentiation was significantly greater during uncertain than certain threats, $\eta_{p}{ }^{2}=.08, b=4.0$, $t(114)=3.10, p=.002$ (Figure 3B). Probe P3 suppression did not significantly differ during uncertain compared to certain threats, $\eta_{p}^{2}=.01, b=0.8, t(121)=1.25, p=.212$ (Figure 3C).

In the no-alcohol participants, subjective anxiety potentiation was significantly greater during uncontrollable than controllable threats, $\eta_{p}^{2}=.49, b=.7, t(115)=10.31, p<.001$ (Figure 4A). Startle potentiation was significantly greater during controllable than uncontrollable threats, $\eta_{p}{ }^{2}=.10, \mathrm{~b}=-4.3, \mathrm{t}(107)=3.44, \mathrm{p}<.001$ (Figure 4B). Probe P3 suppression did not significantly differ during uncontrollable compared to controllable threats, $\eta_{p}^{2}=.00, b=.2, t(121)=.39, p=.700$ (Figure 4C).

Across all stressors, alcohol significantly reduced subjective anxiety potentiation, $\eta_{p}^{2}=.07, b=-.4, t(114)=2.86, p=.005$, startle potentiation, $\eta_{p}^{2}=.18, b=-13.0, t(107)=4.89, p<.001$, and probe P3 suppression, $\eta_{p}{ }^{2}=.06, b=-3.2, t(121)=2.78, p=.006$. We did not observe a significant three-way, alcohol X certainty X controllability interaction for subjective anxiety potentiation, $\eta_{p}{ }^{2}=.01, b=-.1, t(115)=1.11, p=.269$. We observed a significant three-way, alcohol $X$ certainty $X$ controllability interaction for startle potentiation, $\eta_{p}^{2}=.08, b=-7.8, t(107)=3.15$, $p=.002$, but none of the follow-up contrasts were significant (ps >.192). We did not observe a significant three-way, alcohol X certainty X controllability interaction for probe P3 suppression, $\eta_{p}^{2}=.00, b=-0.7, t(121)=.57, p=.568$. 
The alcohol X certainty interaction was significant for subjective anxiety potentiation, such that alcohol's effect on subjective anxiety potentiation was significantly greater during uncertain $\left(\eta_{p}^{2}=.08, b=-.5, t(115)=3.25, p=.002\right)$ than certain threats $\left(\eta_{p}^{2}=.05, b=-0.3\right.$, $t(115)=2.26, p=.026.), \eta_{p}^{2}=.05, b=-0.2, t(115)=2.48, p=.015$ (Figure 3A). The alcohol X certainty interaction was significant for startle potentiation, such that alcohol's effect on startle potentiation was significantly greater during uncertain $\left(\eta_{p}^{2}=.21, b=-15.3, t(107)=5.30, p<.001\right)$ than certain threats $\left(\eta_{p}^{2}=.13, b=-10.7, t(107)=3.92, p<.001\right), \eta_{p}^{2}=.06, b=-4.6, t(107)=2.55$, $p=.012$ (Figure 3B). The alcohol X certainty interaction was not significant for probe P3 suppression, $\eta_{p}{ }^{2}=.03, b=-1.6, t(121)=1.77, p=.080$ (Figure $3 \mathrm{C}$ ).

The alcohol X controllability interaction was not significant for subjective anxiety potentiation, $\eta_{p}^{2}=.00, b=-.1, t(115)=.63, p=.430$ (Figure $4 \mathrm{~A}$ ), startle potentiation, $\eta_{p}^{2}=.03, b=3.2$, $t(107)=1.85, p=.067$ (Figure 4B), or probe P3 suppression, $\eta_{p}^{2}=.00, b=-0.9, t(121)=1.10, p=.275$ (Figure 4C). 

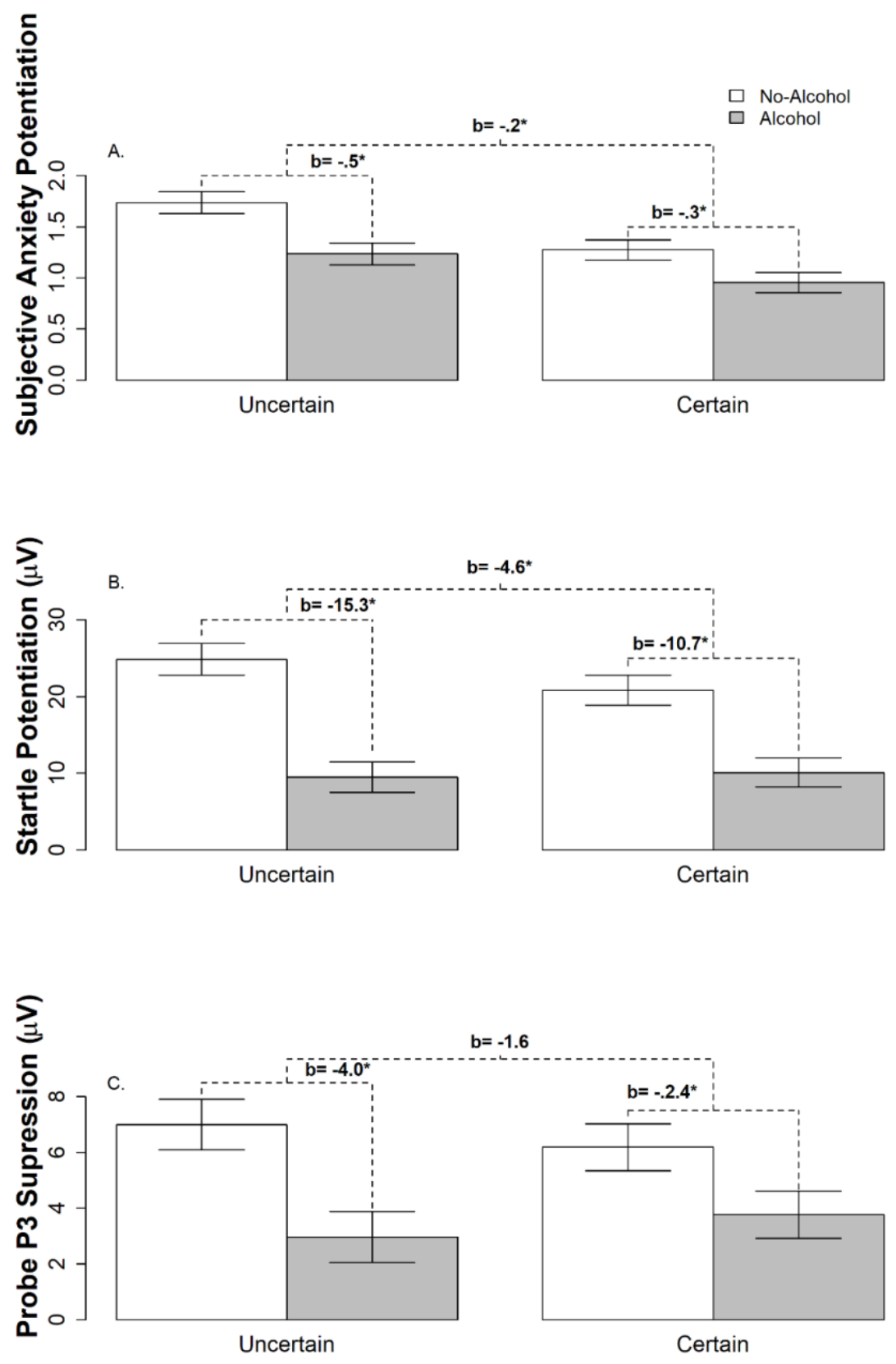

Figure 3: Effects of threat certainty and alcohol for each dependent variable.

Confidence bars represent \pm one standard error for point estimates of each DV from the GLM.

Coefficients from the GLM show the simple effect of Alcohol for each stressor type as well as the stressor type by Alcohol interaction (*p<.05). Figure (C) 2021 Daniel E. Bradford, Jack M. Shireman, Sarah J. Sant'Ana, Gaylen E. Fronk, Susan E. Schneck, John J. Curtin under Creative Commons Attribution 4.0 International Public License CC-By. 

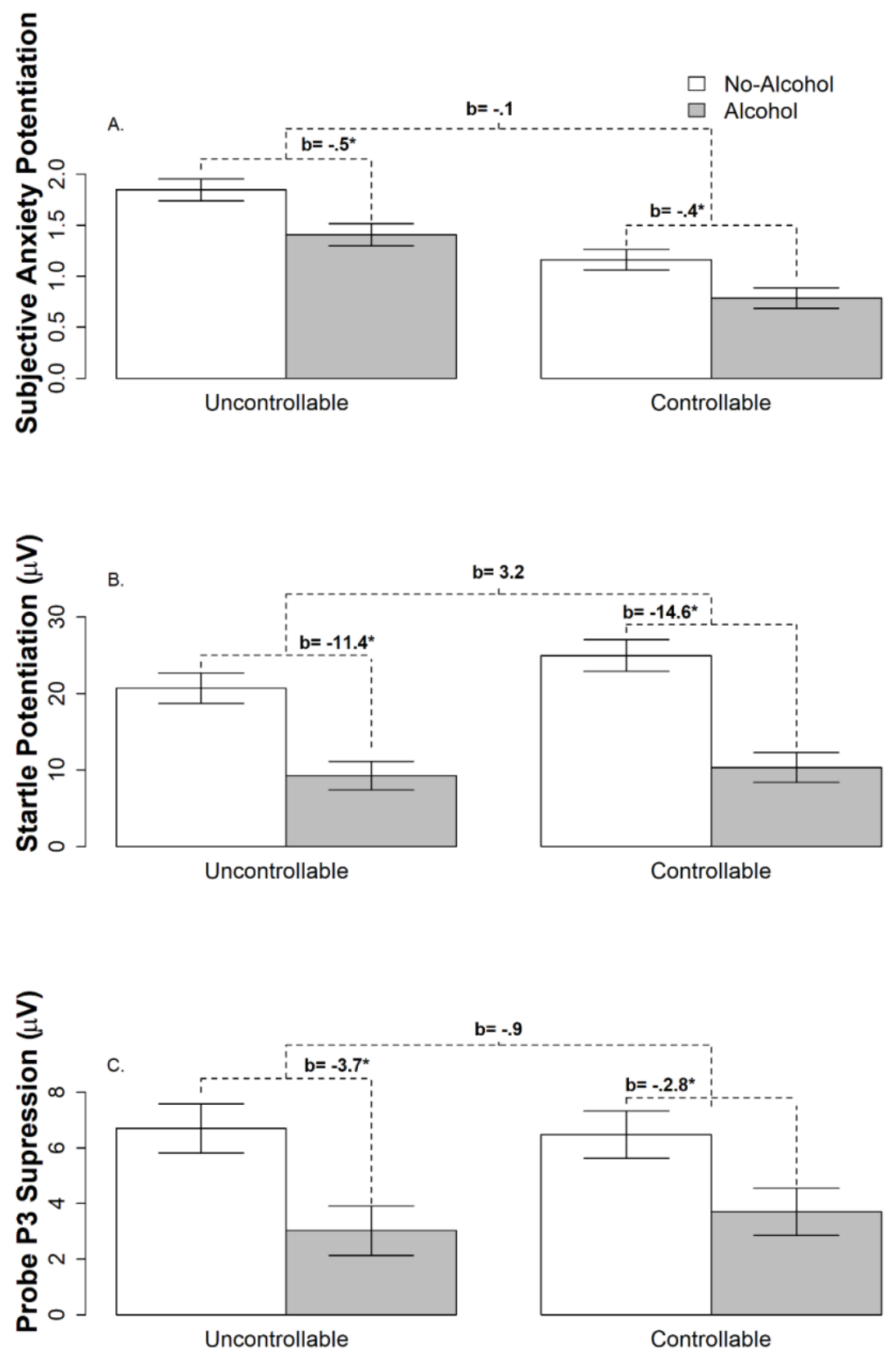

Figure 4: Effects of threat controllability and alcohol for each dependent variable

Confidence bars represent \pm one standard error for point estimates of each DV from the GLM.

Coefficients from the GLM show the simple effect of Alcohol for each stressor type as well as the stressor type by Alcohol interaction (*p<.05). Figure (C) 2021 Daniel E. Bradford, Jack M. Shireman, Sarah J.

Sant'Ana, Gaylen E. Fronk, Susan E. Schneck, John J. Curtin under Creative Commons Attribution 4.0 International Public License CC-By. 


\section{Discussion}

This pre-registered laboratory study rests within a programmatic series of experiments showing evidence that alcohol dampens subjective anxiety and defensive reactivity more during uncertain than certain stressors regardless of the type of uncertainty (e.g., uncertain probability, timing, location, or intensity; Bradford et al., 2017; Kaye et al., 2017)). Like most modern stressor uncertainty research, however, the stressors in previous work were also uncontrollable thus obscuring controllability's influence on the effects of alcohol and uncertainty. Here, we used a novel design to simultaneously manipulate certainty and perceived controllability of stressor intensity. The presence of control significantly affected subjective anxiety and startle potentiation, but alcohol's effects on these responses to uncontrollable versus controllable stressors did not differ. In fact, alcohol appeared to dampen subjective anxiety and startle potentiation more during uncertain than during certain stressors regardless of controllability, potentiality establishing a boundary condition for the enhancement of alcohol's effects on stressor reactivity. The present data suggest that stressor uncertainty — but not uncontrollability — is sufficient to enhance alcohol's stress reactivity dampening and thus negative reinforcement potential.

Though alcohol appears to consistently dampen reactivity to a greater degree during uncertain than certain stressors (Kaye et al., 2017), in the present study, alcohol also dampened reactivity across all response domains, to some degree, regardless of stressor type. As such, the current results may delimit the enhancement of alcohol's effects on stressor reactivity, but not the mere presence of such effects. In fact, alcohol may somewhat dampen reactivity to all types of stressors thus reinforcing use and establishing addictive potential for many stressful situations. In remains unclear, however, how large of a dampening effect on stress reactivity would be 
sufficient to increase risk for addiction. Furthermore, three decades of so-called alcohol Stress Response Dampening (SRD) laboratory research has made clear that alcohol's effects on stress reactivity are often weakly observed, if observed at all (Sayette, 2017; Sher, 1987). The historical inconsistently of discernable alcohol SRD effects has reinforced the need to identify the stressor characteristics that most strongly and reliability predict it. The current results join other recent research (Bradford et al., 2017; Kaye et al., 2017)) to suggest stressor certainty, perhaps more so than other stressor characteristics, is a particularly robust contributor to alcohol SRD.

Our findings may have implications for clarifying motivation for real-world drinking. The present data raise the interesting possibility that drinking to cope with stressors may be particularly reinforcing when those stressors are uncertain (e.g., you worry about the cost of an upcoming bill) regardless of if it is uncontrollable (you think you cannot afford it) or controllable (you think you can afford it). Individuals with a large degree of uncertainty in their lives, such as those with a dearth of social support, separated families or unstable housing may be particularly sensitive to the negative reinforcing effects of alcohol even if they have resources, such as temporary housing or financial assistance, that would allow them to feel some control over those stressors.

Further clarifying the relative roles of certainty and controllability in alcohol's acute and chronic effects on stressor reactivity could potentially inform the neurobiology of substance dependence etiology. Animal models of drug dependence postulate that repeated dampening of the neuropathways involved in response to uncertain stressors by alcohol or other drugs eventually leads to compensatory neuroadaptation that manifests as exaggerated negative affective responses to uncertain stressors that thus motivates further use to reduce negative affect (Kaye et al., 2017; Koob \& Volkow, 2010). Complimentary studies using stress paradigms with 
startle potentiation during uncertain stressors in recreational drinkers coupled with similarly conducted drug deprivation experiments in individuals meeting criteria for drug dependence can provide cross-sectional evidence of the initial effects of drugs as well as the putative consequences of repeated use (see Kaye et al., 2017 for review). Within this framework, the dampening of responses to uncertain stressors seen in the current study could be demonstration of the first stage of a proposed drug induced neuroadaptation to uncertain stressors (Kaye et al., 2017). Preliminary cross-sectional research by our laboratory and others has demonstrated that individuals with alcohol use disorder show exaggerated startle potentiation to uncertain stressors, potentially demonstrating compensatory changes in the consequent stage of these proposed neuroadaptations (Moberg et al., 2017; cf, Gorka et al., 2013; Gorka \& Shankman, 2017). By ruling out the contribution of other stressor characteristics such as controllability, studies such as this one can implicate uncertain stressor reactivity and its associated neural-substrates - rather than exaggerated reactivity broadly or other characteristics with distinct neural-substrates - as key in at least the first stage of these neuroadaptations. Of course, more convincing evidence of these putative neuroadaptations requires a longitudinal approach as could be accomplished in future research by assessing individuals before and after they have substantial exposure to alcohol and other drugs.

The current research joins a growing literature that uses modern experimental methods to assess responses to multiple stressor characteristics to inform understanding of stressor reactivity more broadly. Alcohol's selective effects on uncertain stressor reactivity in humans are consistent with data from translational neuroscience research suggesting reactions to uncertain and certain stressors rely on distinct mechanisms (Davis et al., 2010). Similarly, the finding that alcohol's effects vary for responses to uncertain versus certain, but not uncontrollable versus 
controllable stressors is consistent with research suggesting the mechanisms governing responses to stressor certainty are at least somewhat distinct from those governing responses to stressor controllability (Maier, 2015; Wood et al., 2015). The neuro-circuits and neurotransmitter systems implicated in this research can be further clarified by more direct measurement and /or manipulation using neuroimaging techniques (e.g., fMRI, MEG) coupled with selective neurotransmitter agonists and antagonists and/or methods such as transcranial magnetic stimulation.

While modern laboratory research on stressor reactivity tends to focus on one response domain, the current results demonstrate the importance of using a multi-measure approach to fully characterize stressor reactivity. In this study, subjective anxiety was greater during presentation of uncontrollable compared to controllable stressors, as expected. We observed, unexpectedly, the opposite pattern for startle potentiation. The initial perceived risk of receiving higher intensity shocks (before control was enacted) may have activated neural structures responsible for increased defensive reactivity in the controllable condition at time of startle measurement but before any downstream effects of control. As such, the use of multiple measures of different response domains raises interesting and testable questions about the timing of the neural and psychological mechanisms involved with the processing of stressor controllability. Furthermore, although startle potentiation has long been used to index increased defensive reactivity thought to reflect an increase in emotions such as anxiety, startle is also heavily influenced by attention (Blumenthal, 2015) as startle is lower when attention is focused away from the startle stimulus. While this raises questions about the impact of attention, we could address this by including measurement of probe P3 suppression in the current study. Probe 
P3 suppression did not differ between controllable and uncontrollable stressors which diminishes the likelihood that the results for startle potentiation were due to changes in attention.

Controllable and uncontrollable stressors appeared to demand comparable attentional resources with each capturing attention above the no-shock cues as measured by probe P3 suppression in the no-alcohol participants. This is consistent with recent observations by our laboratory (Bradford et al., 2017) and others (Nelson et al., 2015) of comparable emotionally motivated attention to uncertain and certain stressors. Although some research has suggested that both uncertain and uncontrollable stressors have detrimental impacts on cognitive performance during complex cognitive tasks (Henderson et al., 2012; Robinson et al., 2013), our data suggest that basic, early attentional processes needed to appraise stressors initially in real-time, remain intact across multiple stressor characteristics including controllability, at least in sober individuals.

Despite differences in self-reported anxiety and defensive reactivity for uncontrollable versus controllable stressors in the no-alcohol group, alcohol did not appear to affect responses to these stressor types differently for any of the response domains studied. This may potentially diminish the apparent importance of stressor controllability, relative to certainty, but future research should affirm this by clarifying remaining ambiguities and increasing the generalizability of the current results. For example, though the alcohol x certainty interaction was significant for self-reported anxiety and startle potentiation, the alcohol $\mathrm{x}$ certainty $\mathrm{x}$ controllability interaction was only statistically significant for startle potentiation. Furthermore, although alcohol had a significantly bigger effect on probe $\mathrm{P} 3$ suppression during uncertain versus certain stressors in one past study (Bradford et al., 2017), this effect was only descriptively similar here. As such, future research should continue to examine the robustness 
and replicability of alcohol's effects on self-reported anxiety, startle potentiation, and emotionally motivated attention to various stressor characteristics with additional indices of emotion and attention (e.g., more granular self-report; eye tracking methods) which aid in interpretation of the results.

Other important contributors to the relationship between drug use and response to stressors were beyond the scope of the current study. For example, theories such as the Appraisal Disruption Model (Sayette, 1993) recognize that drinking often occurs following a stressor rather than prior to it as was the case in the current study. The Appraisal Disruption Model and other theories make specific predictions about the relative timing of stressor appraisal, exposure, and drinking. All these components should be carefully manipulated and tested in future research using paradigms similar to here. Importantly, future research to clarify these and other ambiguities should also strive to include as representative of a sample as possible, in terms of broad demographics and clinically relevant characteristics such as drinking history.

Additional research can also extend and bridge the current results to more ecologically valid and clinically relevant contexts. The use of electric shock stressors in the current study is consistent with our program of research focused on careful translation of animal neuroscience that includes precise testing of stressor features to allow speculation about neural mechanism (Kaye et al., 2017). Future research using similar paradigms with different stressors, however, should further extend the current results to other translational paradigms from animal neuroscience (e.g., light/dark enhanced startle) as well as increase the generalizability of the results with stressors more consistent with common human experiences that include uncertainty (e.g., the Trier Social Stress Test). In fact, growing evidence suggests alcohol reduces negative affect more readily in social contexts when uncertain behavior is exhibited by others (e.g., 
"Social-Attribution Approach"; Fairbairn \& Sayette, 2014) and recent ecological momentary assessment research (EMA) supports the theory that these effects of alcohol may reinforce drinking in social contexts (e.g., Goodman et al., 2018). The current paradigm can be modified to include the presence of drinking partners or other social interactions to assess the contribution of social factors on alcohol stressor certainty effects which was not addressed here. Furthermore, generalizability of the current results can be tested using EMA and mobile psychophysiology to examine responses to uncertain stressors and the effects of drug use in the real world.

More broadly, this study parses the mechanisms involved with two frequently confounded stressor dimensions assessed in various response domains using a novel, multiplemeasurement paradigm, and a powerful pharmacological manipulation. Similar approaches could be used for contemporary efforts to leverage psychophysiological markers towards clearer classification and/or diagnosis across psychopathology. Due to the perceived relevance of exaggerated response to uncertain stressors in anxiety disorders, NIH's Research Domain Criteria (RDoC) prominently features distinctions between certain and uncertain stressors, labeled respectively as "acute fear" and "potential threat ("anxiety")" in the Negative Valence Systems domain (Insel et al., 2010). Current RDoC criteria, however, do not currently speak to the controllability of those uncertain and certain stressors. Furthermore, RDoC does not yet include distinctions between uncontrollable and controllable stressors despite their potential relevance in the etiology of depression (Abramson et al., 1978; Maier \& Watkins, 2005). Paradigms such as described here can be implemented to test for patient differences in reactions to stressor controllability and/or to rule out potential contributions of stressor controllability when differences in reactions to stressor certainty are of primary focus. 
Author Contributions: D.E.B., J.M.S., and S.E.S. developed the study concept and design under the supervision of J.J.C. Testing and data collection were performed by D.E.B., J.M.S. and S.J.S. D.E.B. performed the data analysis and interpretation under the supervision of J.J.C. D.E.B. drafted the paper, and D.E.B., J.M.S., S.E.S, and G.E.F provided critical revisions. All authors approved the final version of the paper for submission.

Acknowledgements: We thank Angelica DeFalco for drafting Figures 1 and 2 during the revision process. 


\section{References}

Abramson, L. Y., Seligman, M. E., \& Teasdale, J. D. (1978). Learned helplessness in humans: Critique and reformulation. Journal of Abnormal Psychology, 87(1), 49-74.

Alius, M. G., Pané-Farré, C. A., Löw, A., \& Hamm, A. O. (2015). Modulation of the blink reflex and P3 component of the startle response during an interoceptive challenge. Psychophysiology, 52(1), 140-148. https://doi.org/10.1111/psyp.12295

Blanchard, D. C., Griebel, G., Pobbe, R., \& Blanchard, R. J. (2011). Risk assessment as an evolved threat detection and analysis process. Neuroscience \& Biobehavioral Reviews, 35(4), 991-998. https://doi.org/10.1016/j.neubiorev.2010.10.016

Blumenthal, T. D. (2015). Presidential Address 2014: The more-or-less interrupting effects of the startle response. Psychophysiology. https://doi.org/10.1111/psyp.12506

Blumenthal, T. D., Cuthbert, B. N., Filion, D. L., Hackley, S., Lipp, O. V., \& van Boxtel, A. (2005). Committee report: Guidelines for human startle eyeblink electromyographic studies. Psychophysiology, 42(1), 1-15. https://doi.org/10.1111/j.14698986.2005.00271.x

Bradford, D. E., Magruder, K. P., Korhumel, R. A., \& Curtin, J. J. (2014). Using the threat probability task to assess anxiety and fear during uncertain and certain threat. Journal of Visualized Experiments: JoVE, 91. https://doi.org/10.3791/51905

Bradford, D. E., Motschman, C. A., Starr, M. J., \& Curtin, J. J. (2017). Alcohol's effects on emotionally motivated attention, defensive reactivity, and subjective anxiety during uncertain threats. Social, Cognitive, and Affective Neuroscience. 
Bradford, D. E., Shapiro, B. L., \& Curtin, J. J. (2013). How bad could it be? Alcohol dampens stress responses to threat of uncertain intensity. Psychological Science, 24(12), 25412549. https://doi.org/10.1177/0956797613499923

Bradford, D. E., Starr, M. J., Shackman, A. J., \& Curtin, J. J. (2015). Empirically based comparisons of the reliability and validity of common quantification approaches for eyeblink startle potentiation in humans. Psychophysiology, 52(12), 1669-1681. https://doi.org/10.1111/psyp.12545

Bradley, M. M., Codispoti, M., \& Lang, P. J. (2006). A multi-process account of startle modulation during affective perception. Psychophysiology, 43(5), 486-497. https://doi.org/10.1111/j.1469-8986.2006.00412.x

Brainard, D. H. (1997). The Psychophysics Toolbox. Spatial Vision, 10(4), 433-436.

Champely, S. (2020). Pwr: Basic Functions for Power Analysis. R package version 1.3-0. (1.3-0) [Computer software]. https://CRAN.R-project.org/package=pwr

Curtin, J. J. (2011). PhysBox: The Psychophysiology toolbox. An open source toolbox for psychophysiological data reduction within EEGLab. http://dionysus.psych.wisc.edu/PhysBox.htm

Curtin, J. J. (2015). ImSupport: Support for Linear Models (2.9.2) [Computer software]. https://cran.r-project.org/web/packages/lmSupport/index.html

Davis, M., Walker, D. L., Miles, L., \& Grillon, C. (2010). Phasic vs sustained fear in rats and humans: Role of the extended amygdala in fear vs anxiety. Neuropsychopharmacology Reviews, 35(1), 105-135. https://doi.org/doi:10.1037/npp.2009 
Delorme, A., \& Makeig, S. (2004). EEGLAB: An open source toolbox for analysis of single-trial EEG dynamics including independent component analysis. Journal of Neuroscience Methods, 134(1), 9-21. https://doi.org/10.1016/j.jneumeth.2003.10.009

Fairbairn, C. E., \& Sayette, M. A. (2014). A social-attributional analysis of alcohol response. Psychological Bulletin, 140(5), 1361-1382. https://doi.org/10.1037/a0037563

Goodman, F. R., Stiksma, M. C., \& Kashdan, T. B. (2018). Social Anxiety and the Quality of Everyday Social Interactions: The Moderating Influence of Alcohol Consumption. Behavior Therapy, 49(3), 373-387. https://doi.org/10/gdkhng

Gorka, S. M., Nelson, B. D., \& Shankman, S. A. (2013). Startle response to unpredictable threat in comorbid panic disorder and alcohol dependence. Drug and Alcohol Dependence, 132(1-2), 216-222. https://doi.org/10.1016/j.drugalcdep.2013.02.003

Gorka, S. M., \& Shankman, S. A. (2017). Preliminary evidence that reactivity to uncertain threat is an endophenotype for alcohol use disorder. Drug \& Alcohol Dependence, 180, 265271. https://doi.org/10.1016/j.drugalcdep.2017.08.023

Greeley, J., \& Oei, T. (1999). Alcohol and tension reduction. In K. E. Leonard \& H. T. Blane (Eds.), Psychological theories of drinking and alcoholism (2nd ed., pp. 14-53). The Guilford Press.

Grillon, C. (2008). Models and mechanisms of anxiety: Evidence from startle studies. Psychopharmacology, 199(3), 421-437. https://doi.org/10.1007/s00213-007-1019-1

Grillon, C., Baas, J. M., Pine, D. S., Lissek, S., Lawley, M., Ellis, V., \& Levine, J. (2006). The benzodiazepine alprazolam dissociates contextual fear from cued fear in humans as assessed by fear-potentiated startle. Biological Psychiatry, 60(7), 760-766. https://doi.org/10/1016/j.biopsych.2005.11.027 
Grillon, C., Chavis, C., Covington, M. F., \& Pine, D. S. (2009). Two-week treatment with the selective serotonin reuptake inhibitor citalopram reduces contextual anxiety but not cued fear in healthy volunteers: A fear-potentiated startle study. Neuropsychopharmacology, 34(4), 964-971. https://doi.org/10.1038/npp.2008.141

Grillon, C., Lissek, S., Rabin, S., McDowell, D., Dvir, S., \& Pine, D. S. (2008). Increased anxiety during anticipation of unpredictable but not predictable aversive stimuli as a psychophysiologic marker of panic disorder. American Journal of Psychiatry, 165(7), 898-904. https://doi.org/10.1176/appi.ajp.2007.07101581

Grillon, C., Pine, D. S., Lissek, S., Rabin, S., Bonne, O., \& Vythilingam, M. (2009). Increased anxiety during anticipation of unpredictable aversive stimuli in posttraumatic stress disorder but not in generalized anxiety disorder. Biological Psychiatry, 66(1), 47-53. https://doi.org/10.1016/j.biopsych.2008.12.028

Hanson, D. J. (2013). Historical evolution of alcohol consumption in society. Oxford University Press. http://www.oxfordscholarship.com/view/10.1093/acprof:oso/9780199655786.001.0001/a cprof-9780199655786-chapter-01

Havranek, M. M., Bolliger, B., Roos, S., Pryce, C. R., Quednow, B. B., \& Seifritz, E. (2016). Uncontrollable and unpredictable stress interacts with subclinical depression and anxiety scores in determining anxiety response. Stress (Amsterdam, Netherlands), 19(1), 53-62. https://doi.org/10.3109/10253890.2015.1117449

Hefner, K. R., \& Curtin, J. J. (2012). Alcohol stress response dampening: Selective reduction of anxiety in the face of uncertain threat. Journal of Psychopharmacology (Oxford, England), 26(2), 232-244. https://doi.org/10.1177/0269881111416691 
Hefner, K. R., Moberg, C. A., Hachiya, L. Y., \& Curtin, J. J. (2013). Alcohol stress response dampening during imminent versus distal, uncertain threat. Journal of Abnormal Psychology, 122(3), 756-769. https://doi.org/10.1037/a0033407

Hefner, K. R., Starr, M. J., \& Curtin, J. J. (2018). Heavy marijuana use but not deprivation is associated with increased stressor reactivity. Journal of Abnormal Psychology, 127(4), 348-358. https://doi.org/10.1037/abn0000344

Henderson, R. K., Snyder, H. R., Gupta, T., \& Banich, M. T. (2012). When does stress help or harm? The effects of stress controllability and subjective stress response on stroop performance. Frontiers in Psychology, 3, 179. https://doi.org/10.3389/fpsyg.2012.00179

Hogle, J. M., Kaye, J. T., \& Curtin, J. J. (2010). Nicotine withdrawal increases threat-induced anxiety but not fear: Neuroadaptation in human addiction. Biological Psychiatry, 68(8), 687-688. https://doi.org/10.1016/j.biopsych.2010.06.003

Hurlbut, S. C., \& Sher, K. J. (1992). Assessing alcohol problems in college students. Journal of American College Health., 41(2), 49-58.

Insel, T., Cuthbert, B., Garvey, M., Heinssen, R., Pine, D., Quinn, K., Sanislow, C., \& Wang, P. (2010). Research domain criteria (RDoC): Toward a new classification framework for research on mental disorders. The American Journal of Psychiatry, 167(7), 748-751. https://doi.org/10.1176/appi.ajp.2010.09091379

Kaye, J. T., Bradford, D. E., Magruder, K. P., \& Curtin, J. J. (2017). Probing for Neuroadaptations to Unpredictable Stressors in Addiction: Translational Methods and Emerging Evidence. Journal of Studies on Alcohol and Drugs, 78(3), 353-371. https://doi.org/10.15288/jsad.2017.78.353 
Koob, G. F., \& Volkow, N. D. (2010). Neurocircuitry of addiction. Neuropsychopharmacology Reviews, 35(1), 217-238. https://doi.org/10.1038/npp.2009.110

Koolhaas, J. M., Bartolomucci, A., Buwalda, B., de Boer, S. F., Flügge, G., Korte, S. M., Meerlo, P., Murison, R., Olivier, B., Palanza, P., Richter-Levin, G., Sgoifo, A., Steimer, T., Stiedl, O., van Dijk, G., Wöhr, M., \& Fuchs, E. (2011). Stress revisited: A critical evaluation of the stress concept. Neuroscience \& Biobehavioral Reviews, 35(5), 12911301. https://doi.org/10.1016/j.neubiorev.2011.02.003

Linden, D. E. J. (2005). The p300: Where in the brain is it produced and what does it tell us? The Neuroscientist: A Review Journal Bringing Neurobiology, Neurology and Psychiatry, 11(6), 563-576. https://doi.org/10.1177/1073858405280524

Liu, R. T., Kleiman, E. M., Nestor, B. A., \& Cheek, S. M. (2015). The Hopelessness Theory of Depression: A Quarter Century in Review. Clinical Psychology: A Publication of the Division of Clinical Psychology of the American Psychological Association, 22(4), 345365. https://doi.org/10/gg887w

Löw, A., Weymar, M., \& Hamm, A. O. (2015). When Threat Is Near, Get Out of Here: Dynamics of Defensive Behavior During Freezing and Active Avoidance. Psychological Science. https://doi.org/10.1177/0956797615597332

Maier, S. F. (2015). Behavioral control blunts reactions to contemporaneous and future adverse events: Medial prefrontal cortex plasticity and a corticostriatal network. Neurobiology of Stress, 1, 12-22. https://doi.org/10.1016/j.ynstr.2014.09.003

Maier, S. F., \& Watkins, L. R. (2005). Stressor controllability and learned helplessness: The roles of the dorsal raphe nucleus, serotonin, and corticotropin-releasing factor. 
Neuroscience and Biobehavioral Reviews, 29(4-5), 829-841.

https://doi.org/10.1016/j.neubiorev.2005.03.021

MATLAB and Statistics Toolbox. (2013). The Mathworks, Inc.

Mineka, S., \& Kihlstrom, J. F. (1978). Unpredictable and Uncontrollable Events: A New Perspective on Experimental Neurosis. Journal of Abnormal Psychology, 87(2), 256-271.

Moberg, C. A., Bradford, D. E., Kaye, J. T., \& Curtin, J. J. (2017). Increased startle potentiation to unpredictable stressors in alcohol dependence: Possible stress neuroadaptation in humans. Journal of Abnormal Psychology, 126(4), 441-453. https://doi.org/10.1037/abn0000265

Moberg, C. A., \& Curtin, J. J. (2009). Alcohol selectively reduces anxiety but not fear: Startle response during unpredictable vs. predictable threat. Journal of Abnormal Psychology, 118(2), 335-347. https://doi.org/10.1037/a0015636

Moberg, C. A., Weber, S., \& Curtin, J. J. (2011). Alcohol dose effects on stress response to cued threat vary by threat intensity. Psychopharmacology, 218, 217-227.

Nelson, B. D., \& Hajcak, G. (2017). Defensive motivation and attention in anticipation of different types of predictable and unpredictable threat: A startle and event-related potential investigation. Psychophysiology. https://doi.org/10.1111/psyp.12869

Nelson, B. D., Hajcak, G., \& Shankman, S. A. (2015). Event-related potentials to acoustic startle probes during the anticipation of predictable and unpredictable threat. Psychophysiology, 52(7), 887-894. https://doi.org/10.1111/psyp.12418

Pelli, D. G. (1997). The VideoToolbox software for visual psychophysics: Transforming numbers into movies. Spatial Vision, 10(4), 437-442. 
Picton, T. W., Bentin, S., Berg, P., Donchin, E., Hillyard, S. A., Johnson, R., Miller, G. A., Ritter, W., Ruchkin, D. S., Rugg, M. D., \& Taylor, M. J. (2000). Guidelines for using human event-related potentials to study cognition: Recording standards and publication criteria. Psychophysiology, 37(2), 127-152.

R Development Core Team. (2015). R: A language and environment for statistical computing. $\mathrm{R}$ Foundation for Statistical Computing. http://www.R-project.org

Robinson, O. J., Bond, R. L., \& Roiser, J. P. (2015). The impact of threat of shock on the framing effect and temporal discounting: Executive functions unperturbed by acute stress? Frontiers in Psychology, 6, 1315. https://doi.org/10.3389/fpsyg.2015.01315

Robinson, O. J., Vytal, K., Cornwell, B. R., \& Grillon, C. (2013). The impact of anxiety upon cognition: Perspectives from human threat of shock studies. Frontiers in Human Neuroscience, 7. https://doi.org/10.3389/fnhum.2013.00203

RStudio Team. (2016). RStudio: Integrated Development for R. RStudio, Inc. http://www.rstudio.com/

Sayette, M. A. (1993). An appraisal-disruption model of alcohol's effects on stress responses in social drinkers. Psychological Bulletin, 114(3), 459-476.

Sayette, M. A. (2017). The effects of alcohol on emotion in social drinkers. Behaviour Research and Therapy, 88, 76-89. https://doi.org/10.1016/j.brat.2016.06.005

Schönbrodt, F. D., Maier, M., Heene, M., \& Zehetleitner, M. (2015). Voluntary commitment to research transparency. http://www.researchtransparency.org

Sher, K. J. (1987). Stress response dampening. In H. T. Blane \& K. E. Leonard (Eds.), Psychological Theories of Drinking and Alcoholism (pp. 227-271). Guilford Press. 
Simmons, J. P., Nelson, L. D., \& Simonsohn, U. (2012, October 14). A 21 Word Solution [SSRN Scholarly Paper]. http://dx.doi.org/10.2139/ssrn.2160588

Testa, M., Fillmore, M. T., Norris, J., Abbey, A., Curtin, J. J., Leonard, K. E., Mariano, K. A., Thomas, M. C., Nomensen, K. J., George, W. H., VanZile-Tamsen, C., Livingston, J. A., Saenz, C., Buck, P. O., Zawacki, T., Parkhill, M. R., Jacques, A. J., \& Hayman Jr., L. W. (2006). Understanding alcohol expectancy effects: Revisiting the placebo condition. Alcoholism: Clinical and Experimental Research, 30(2).

Wendt, J., Löw, A., Weymar, M., Lotze, M., \& Hamm, A. O. (2017). Active avoidance and attentive freezing in the face of approaching threat. NeuroImage, 158, 196-204. https://doi.org/10.1016/j.neuroimage.2017.06.054

Wood, K. H., Wheelock, M. D., Shumen, J. R., Bowen, K. H., Ver Hoef, L. W., \& Knight, D. C. (2015). Controllability modulates the neural response to predictable but not unpredictable threat in humans. NeuroImage, 119, 371-381. https://doi.org/10.1016/j.neuroimage.2015.06.086

Zvolensky, M. J., Lejuez, C. W., \& Eifert, G. H. (2000). Prediction and control: Operational definitions for the experimental analysis of anxiety. Behaviour Research and Therapy, $38(7), 653-663$. 